\title{
Breakdown delay times and memory effects in helium at low pressure
}

\author{
V Kudrle $\dagger$, E LeDuc and M Fitaire \\ Laboratoire de Physique des Gaz et des Plasmas, Bâtiment 210, Université Paris-Sud, \\ 91405 Orsay Cedex, France
}

Received 15 December 1998, in final form 29 March 1999

\begin{abstract}
When the series of voltage pulses is applied to a gas-filled discharge tube, one observes that a breakdown and the subsequent discharge will influence the probability of the next breakdown. Repeating the measurement of the breakdown delay time $\tau$ many times we can determine the distribution of $\tau$ and subsequently decompose breakdown delay time into a formative time lag and the statistical delay time. Our measurements show that even a measurement taken $10 \mathrm{~min}$ after the last breakdown is influenced by it. The processes in a volume being unimportant due to a flow mode of operation, it is proposed that the memory effect is caused by some surface processes. The experiments are carried out in a discharge tube with two plane-parallel electrodes separated by $2 \mathrm{~cm}$ in a pressure range of several Torr. In this paper, dependences of both statistical delay time and formative time lag on the repetition rate, discharge duration and anode voltage are presented.
\end{abstract}

\section{Introduction}

The time required for an electrical discharge to establish itself after the application of a voltage is of considerable scientific and practical interest. Rapid discharge switches are used in many applications. Often low delay and jitter are considered as very important [1], and thus they are decreased by the presence of some ionizing agent. The lifetime of active particles in the afterglow is another interesting problem. For example, the proper function of high-voltage devices, used in power applications, depends on the time needed for restoration of the dielectric strength of a gap after the breakdown.

Electrical breakdown of a gas has been known for a long time. Investigators observed early on that the delay between an application of voltage to the electrodes and the establishment of a self-consistent discharge has a strong stochastic character. Two fundamental conditions must be satisfied for the breakdown to occur: at least one free electron should be present in the interelectrode gap and the electric field should be sufficiently high to multiply the electrons. Several theoretical studies [2-7] of the initial stage of a breakdown have been developed, taking into account the nondeterministic nature of both these phenomena.

Generally the breakdown delay time consists of two components: the statistical delay time $t_{\mathrm{s}}$, when the first electron appears, and the formative time lag $t_{\mathrm{f}}$, necessary to develop the discharge. Naturally, when there are more electrons present at the beginning, there is a greater chance that one of them will initiate the discharge. Also the

$\dagger$ On leave from Department of Physical Electronics, Masaryk University, CZ 61137 Brno, Czech Republic. development of a discharge will be faster. When the number of initial electrons is very large, qualitatively different phenomena may occur.

The initial electrons are produced by natural causes, like cosmic rays or the radioactive background of the Earth, or by artificial ionizing agents, most often the UV illumination of the cathode. Free electrons and/or the active particles, capable of producing them, may also rest in a discharge volume after the discharge is switched off. Their number decreases with time but in comparison with the natural creation rate $\left(10 \mathrm{e}^{-}\left(\mathrm{s} \mathrm{cm}^{3}\right)^{-1}\right.$ in air at atmospheric pressure [8]) they may still be of a great importance in the initiation of a breakdown. The memory effect, the long-time variation of breakdown delay time $\tau$ as a function of the parameters of the preceding discharge, has been observed for a number of different cathode materials, several gases and various experimental set-ups [9-19].

In this study we investigate the influence of products of a low-pressure helium glow discharge on the next breakdown. By means of a statistical treatment, $t_{\mathrm{s}}$ and $t_{\mathrm{f}}$ are extracted from experimental data and their dependence on several experimental parameters is presented. We discuss the possible causes of the observed long-time memory effects and some practical suggestions on how to deal with them are given (the method of interlaced pulses, measurement of the breakdown voltage).

\section{Time lags in breakdown experiments}

The mean statistical delay time is inversely proportional to the electron creation rate. We must also include the probability that an initial electron gives rise to an infinite number of 
V Kudrle et al

avalanches. In the one-dimensional case, it depends on the distance $x$ from the cathode, in which the seed electron appeared

$$
\frac{1}{t_{\mathrm{s}}}=\int_{0}^{d} j(x) P(x) \mathrm{d} x
$$

where $j(x)$ is the spatial density of the number of electrons created in a gap per second, $P(x)$ is the probability that a discharge will develop from the initial electron in position $x$ and $d$ is the distance of the electrodes. We may formally rewrite this equation in the form

$$
t_{\mathrm{s}}=\frac{1}{I_{0} P_{0}}
$$

where $I_{0}$ is the total number of created electrons per unit time and $P_{0}$ is some mean value of $P(x)$.

Let $Q(t)$ be the probability that no breakdown will occur in the interval $(0 ; t)$. It may easily be shown that

$$
Q(t)= \begin{cases}1 & \text { for } t \leqslant t_{\mathrm{f}} \\ \exp \left[\int_{0}^{t-t_{\mathrm{f}}}-I_{0}\left(t^{\prime}\right) P_{0}\left(t^{\prime}\right) \mathrm{d} t^{\prime}\right] & \text { for } t>t_{\mathrm{f}} .\end{cases}
$$

Under the condition $I_{0} P_{0} \neq f(t)$ this implies

$$
Q(t)=\exp \left(-\frac{t-t_{\mathrm{f}}}{t_{\mathrm{s}}}\right) .
$$

From a series of measurements with fixed $I_{0} P_{0}$ we obtain a distribution $\rho(\tau)$ of the breakdown delay time. From the definition of $Q(t)$ we have

$$
Q(t)=1-\int_{0}^{t} \rho(\tau) \mathrm{d} \tau .
$$

Now, if a logarithm of $Q(t)$ is plotted versus time, we should observe a plateau, corresponding to times shorter than $t_{\mathrm{f}}$ $\left(Q\left(t<t_{\mathrm{f}}=1\right)\right)$ and a linear decrease for $t>t_{\mathrm{f}}$. From the slope of this decrease, statistical delay time is calculated.

\section{Apparatus}

A schematic drawing of the experimental arrangement is shown in figure 1 . Two circular $(1.4 \mathrm{~cm}$ diameter) electrodes made of technical aluminium are placed in a Pyrex discharge tube with $2 \mathrm{~cm}$ inner diameter. The interelectrode distance is $d=2 \mathrm{~cm}$. The flow of working gas (AirLiquide Helium C) through the discharge tube is kept constant using a mass flow controller FC. The pressure is measured with a capacitive gauge and set by a throttling valve TV between the tube and a rotary oil pump RV. All measurements are made at room temperature $(\approx 300 \mathrm{~K})$. The electronics and the discharge tube are placed in a metal box and protected from the light.

The electrodes were polished before installation by fine abrasive paper and cleaned in ethylalcohol. With Viton seals being used, no baking was performed, but only a heating to $100^{\circ} \mathrm{C}$ over several days. The system was pumped permanently down to several $10^{-3}$ Torr when no measurements were performed.

The manufacturer of technical grade gas states the abundances of $\mathrm{H}_{2} \mathrm{O}, \mathrm{O}_{2}$ and $\mathrm{C}_{x} \mathrm{H}_{y}$ impurities to be lower than

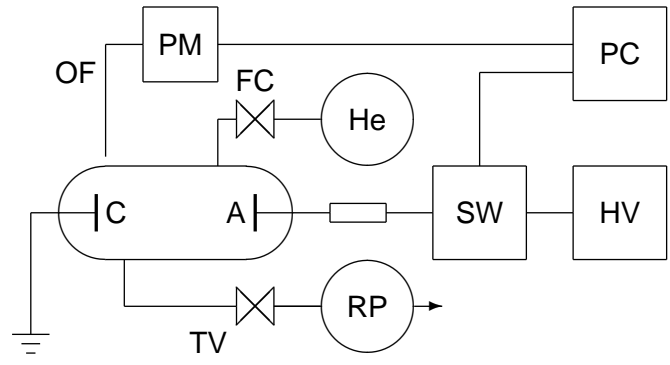

Figure 1. Experimental set-up

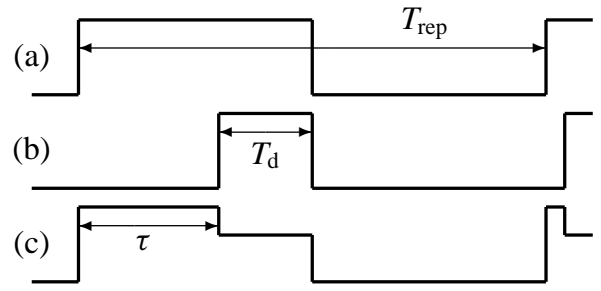

Figure 2. A schematic drawing of signals: (a) switch command; (b) discharge current; (c) voltage on anode.

3 ppm, but a small admixture $(0.01 \%)$ of neon is permitted. We controlled the composition of the gas flowing through the discharge tube by a quadrupole mass spectrometer and we found that the total contamination by air (through possible leaks and desorption) was not greater than $0.1 \%$. The abundance of hydrocarbons (due to possible backstreaming from the pump) was under $100 \mathrm{ppm}$.

The cathode is grounded and the anode is driven by a stabilized high-voltage supply HV through a load resistance and a fast (computer controlled) solid-state, high-voltage switch SW. The rise time $t_{\mathrm{on}}=1.5 \mu \mathrm{s}$ of the anode voltage pulse is determined mostly by the stray capacities $(C \approx$ $\left.10^{1} \mathrm{pF}\right)$ and the load resistor $(R=56 \mathrm{k} \Omega)$. Onset of the discharge is detected using an optic fibre OF and a photomultiplier PM (Hamamatsu 928). The time interval $\tau$ between the rising edge of the voltage on the anode and the electrical breakdown of the gas is measured with a precision of $1 \mu \mathrm{s}$ by means of an electronic counter (IC AMD9513).

A series of high-voltage pulses with period $T_{\text {rep }}$ and duration $T_{\mathrm{a}}$ is applied to the anode (see figure 2). At the instant $t=0$ the anode voltage is switched on and the counter is started. After some random time an electron appears in the interelectrode space. Being accelerated by the electric field, the electron can give rise to a series of consecutively growing electronic avalanches. The atoms, excited by electronic collisions, start to emit photons. Some of these photons are then captured by a photomultiplier which outputs a current proportional to the photon flux. When the photocurrent reaches a preset value, the counter is stopped and its value is read by the computer PC. The discharge is switched off after a predefined period $T_{\mathrm{d}}$. When a breakdown does not occur before maximum anode pulse width $T_{\mathrm{a}}$, the non-breakdown event is saved. To obtain statistically significant data, the measurements are repeated many times $\left(N \approx 10^{2}-10^{3}\right)$ with fixed period $T_{\text {rep }}$. 

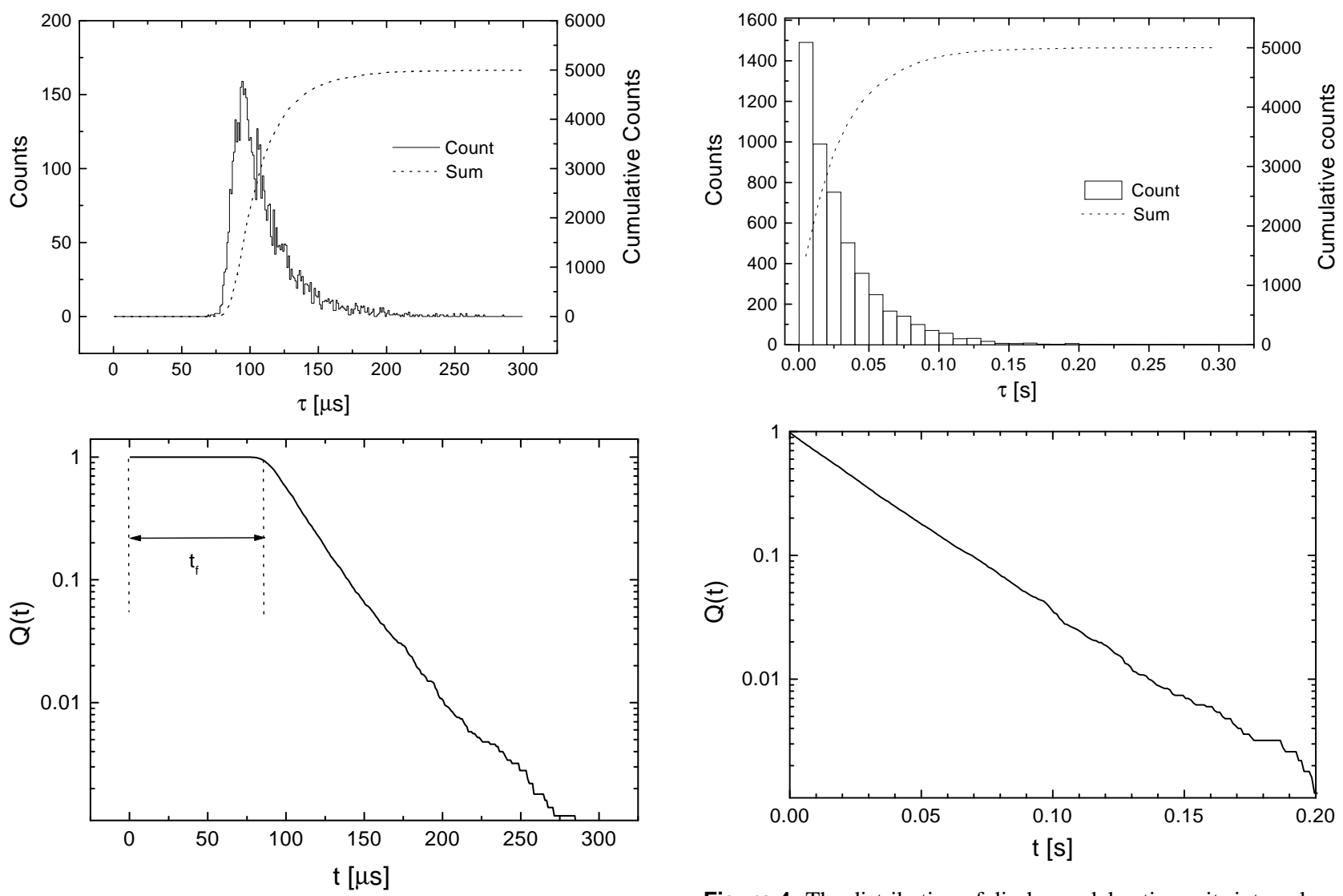

Figure 3. The distribution of discharge delay times, its integral and derived probability $Q(t)$. A series of 5000 measurements was performed under the conditions: $p=4$ Torr, $U=600 \mathrm{~V}$, $T_{\text {rep }}=10 \mathrm{~s}$ and $T_{\mathrm{d}}=800 \mu \mathrm{s}$.

\section{Results and discussion}

Plots of $Q(t)$ on a semi-logarithmic scale yield straight lines in accordance with the assumption that $I_{0} P_{0}$ is constant during the period $T_{\mathrm{a}}$. We present in figure 3 the distribution of breakdown delay times obtained from a series of 5000 measurements for voltage $U=600 \mathrm{~V}$, repetition rate $T_{\text {rep }}=10 \mathrm{~s}$, discharge duration $T_{\mathrm{d}}=800 \mu \mathrm{s}$ and pressure $p=4$ Torr. One observes that no breakdowns occur earlier than $70 \mu \mathrm{s}$ after the application of the high voltage. The decay from $100 \mu$ s may be directly fitted by an exponential to obtain a value of the statistical delay time. A better way is to construct an integral, which is subjected to statistical fluctuations to a much lesser extent. After normalization to the number of measurements, the integral is subtracted from the unity (see equation (5)) and the resulting curve of $Q(t)$ is found. Using a method of least squares, the values $t_{\mathrm{f}}=83.7 \mu \mathrm{s}$ and $t_{\mathrm{s}}=37.6 \mu \mathrm{s}$ were calculated. The difference between the minimal breakdown delay time and $t_{\mathrm{f}}$ determined from $Q(t)$ curve can be attributed [20] to the possible variations of $t_{\mathrm{f}}$.

When the number of active particles at the instant of application of the high voltage on the anode is low, the statistical delay time $t_{\mathrm{s}}$ becomes much greater than the formative time lag $t_{\mathrm{f}}$. This can by achieved by reducing the discharge duration or by increasing the $T_{\text {rep }}$. Figure 4 presents the results of 5000 measurements under the same

Figure 4. The distribution of discharge delay times, its integral and derived probability $Q(t)$. A series of 5000 measurements was performed under the conditions: $p=4$ Torr, $U=600 \mathrm{~V}$, $T_{\text {rep }}=10 \mathrm{~s}$ and $T_{\mathrm{d}}=50 \mu \mathrm{s}$.

conditions as in figure 3, except for the discharge duration, which is reduced to $T_{\mathrm{d}}=50 \mu \mathrm{s}$. The time of development of a discharge is negligible in comparison with the statistical fluctuations of the instant of arrival of the first electron. A plot of $Q(t)$ yields a straight line as before but the plateau, which corresponds to $t_{\mathrm{f}}$, is unobservable. From the slope we can determine $t_{\mathrm{s}}=28.3 \mathrm{~ms}$.

Qualitatively, the same behaviour is observed when the number of residual active particles is reduced by decreasing the voltage (and current) of a discharge or due to the longer periods between the measurements.

The memory curves (the dependence of breakdown delay time on repetition rate) are presented in figure 5 for three different voltages over a range of time intervals $T_{\text {rep }}$ from 10 to $600 \mathrm{~s}$. The points yield straight lines on a log-log plot and their slope is independent of voltage. If we consider a process of first order responsible for the active particle loss and creation of free electrons we should observe an exponential decay of the concentration of active particles, which is not true in our case. The emission of electrons due to the surface space charge on isolating cathode layers, being a first-order process, should thus be rejected as a long-lived post emission source.

For a second-order process, both the loss of active particles and the creation of electrons are proportional to the square of the active particle concentration

$$
I_{0}=k_{1} N^{2}
$$




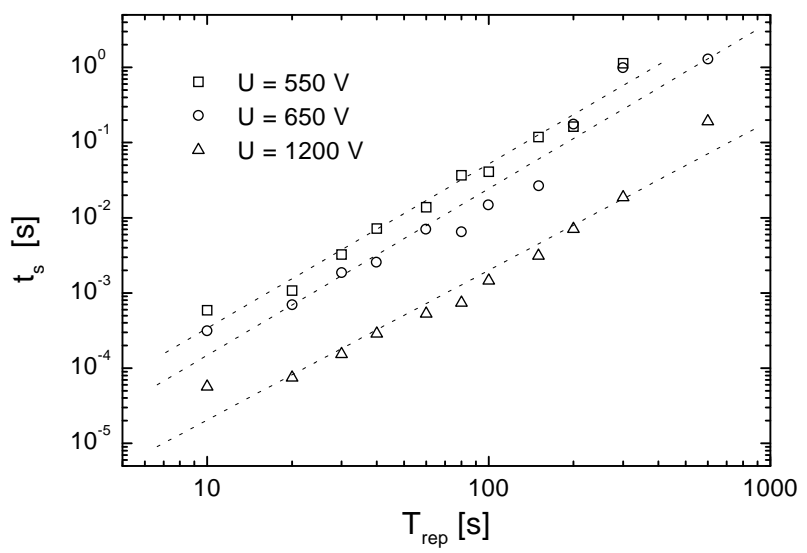

Figure 5. Statistical delay time as a function of repetition rate for $p=8$ Torr and $T_{\mathrm{d}}=20 \mu \mathrm{s}$.

$$
\frac{\mathrm{d} N}{\mathrm{~d} t}=-k_{2} N^{2}
$$

where $k_{1}$ and $k_{2}$ are rate coefficients. This leads to a characteristic decay

$$
\frac{1}{N}=\frac{1}{N_{0}}+k_{2} t
$$

The breakdown delay time is inversely proportional to $I_{0}$ and thus we obtain [14]

$$
\sqrt{t_{\mathrm{s}}}=\sqrt{t_{\mathrm{s} 0}}+\frac{k_{2}}{\sqrt{k_{1} P_{0}}} t .
$$

When we put the square root of the statistical delay time $\sqrt{t_{\mathrm{s}}}$ on the ordinate, its dependence on the period $T_{\text {rep }}$ is linear (figure 6). A typical case of a second-order process is volume recombination. In our case, the flow regime of the experiments prevents the volume processes having a substantial influence on the breakdown. Similarly, the long-lived metastables may also be rejected as a possible source of secondary electrons, because the quenching of such states by collisions with other atoms reduces their lifetime to far lower values than we actually observed. Moreover, a flow of working gas through the tube should quickly remove any remaining products of a discharge. The interelectrode volume $\left(V=3.1 \mathrm{~cm}^{3}\right)$ is at pressure 8 Torr and gas flow $30 \mathrm{sccm}$, replaced after $65 \mathrm{~ms}$. We thus conclude that secondorder recombination at the cathode surface is the main cause of the observed memory effects.

The memory effect may propagate to the next series, as shown in figure 7 , where the result of eight consecutive series, without any delay between them, with varying $T_{\text {rep }}$, is plotted. As awaited, the breakdown delay time rises when the period of inactivity between two measurements is increased. However, the measurements from the beginning of the series maintain the previous value of $\tau$. After the end of one series, the system needs some time to find its new equilibrium. Closer examination yields the that number of breakdowns and not the time seems to be the main factor for equilibrium re-establishment. It is clear that breakdown $i$ is not only influenced by the discharge associated with breakdown $i-1$, but by many more preceding breakdowns.

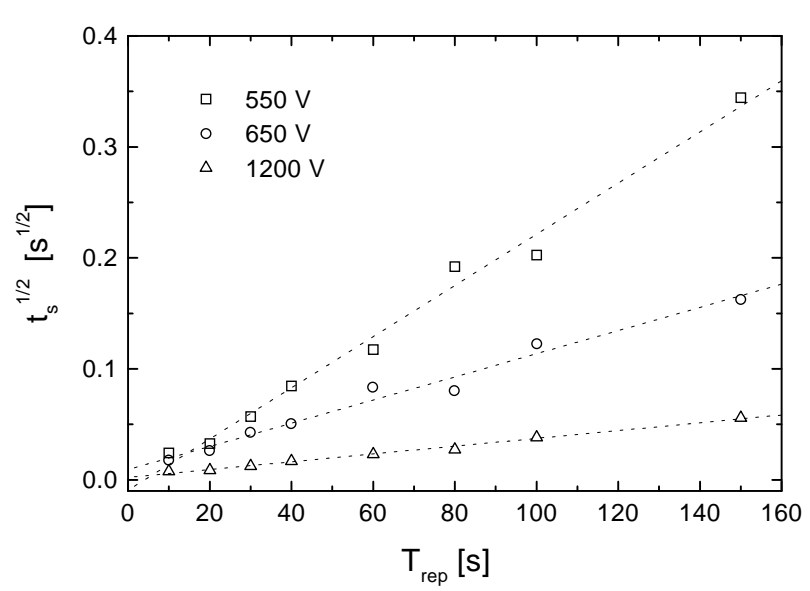

Figure 6. The square root of the statistical delay time as a function of repetition rate. The data with sufficiently high statistical weight, i.e. a great number of measurements, are taken from figure 5 . For higher values of $T_{\text {rep }}$ the data points have a greater error due to an increasing number of non-breakdown events.

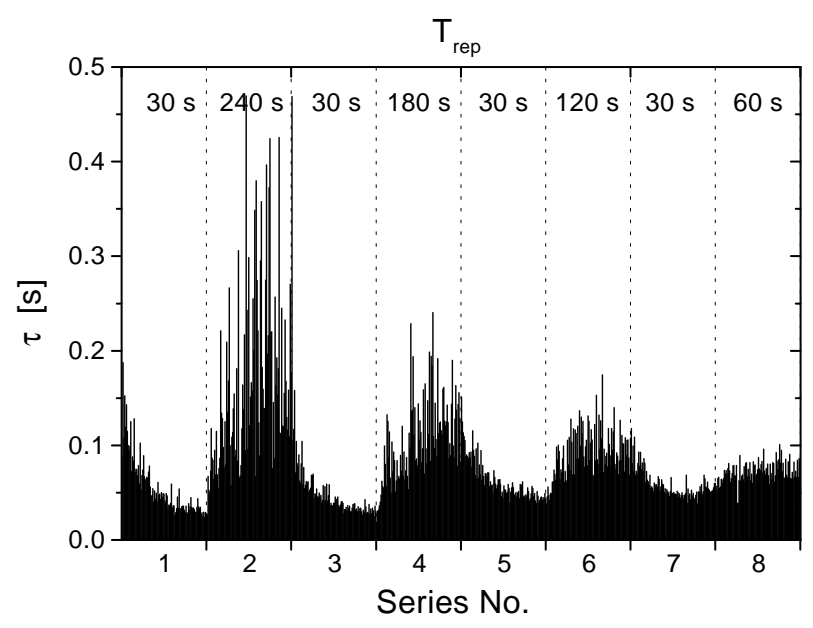

Figure 7. Evolution of breakdown delay times during eight consecutive series, each with 100 measurements. The anode voltage $U=350 \mathrm{~V}$, helium pressure $p=3$ Torr and discharge duration $T_{\mathrm{d}}=20 \mu \mathrm{s}$.

Therefore, special precautions must be taken when reproducibility is important. As a good practice, several tens of breakdowns at given experimental conditions should be carried out before the series. Only then can the tube be considered as conditioned and its properties should not change during the measurements.

The fact that hysteresis exists in both directions (increasing and decreasing $T_{\text {rep}}$ ) suggests that conditions providing the 'memory' electrons are reproduced during the discharge operation. The number of active particles created by a discharge is surely greater than the number of these particles, residing from the previous discharge. It seems that, besides changes of $N$, some slower process that changes the efficiency of transformation of the active particles to free electrons or changes the probability $P_{0}$ takes place. Among possible explanations, the processes changing the cathode surface physically or chemically are the most probable.

The mean breakdown delay time as a function of both $T_{\mathrm{d}}$ and $T_{\text {rep }}$ is presented in figure 8 . No visible changes of 


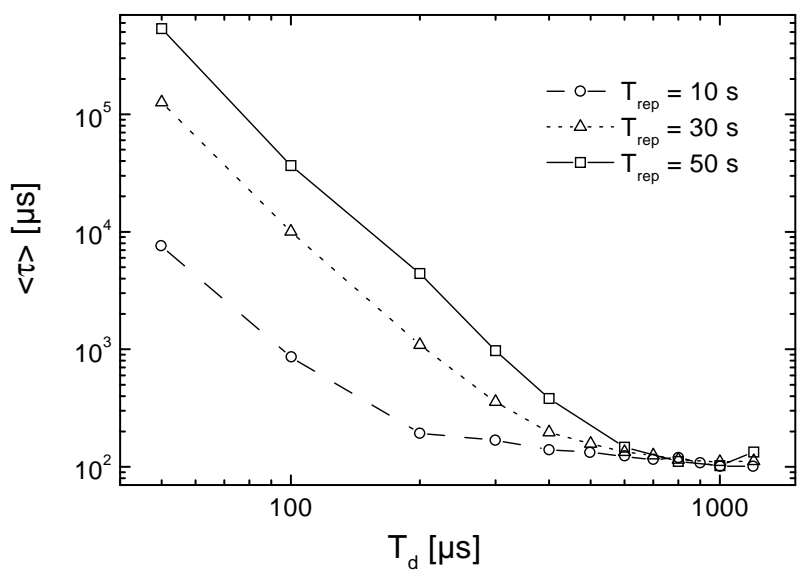

Figure 8. Mean breakdown delay time $\langle\tau\rangle$ as a function of $T_{\mathrm{d}}$ for three values of $T_{\text {rep }}$. The measurements are made at $100 \%$ overvoltage $(U=600 \mathrm{~V})$ and pressure $p=4$ Torr.

appearance of the discharge (diffusive glow without cathode spots) were observed when varying $T_{\mathrm{d}}$. For long discharge durations the number of residual active particles is so high that the breakdown delay time is approaching a limit- the formative time lag. The points at shorter $T_{\mathrm{d}}$ tend to form a straight line with a slope of -3.33 . Considering a secondorder process, we obtain

$$
N \propto T_{\mathrm{d}}^{5 / 3}
$$

The fact that both $I_{0}$ and $P_{0}$ are functions of voltage is a complication in the studies of the dependence of $t_{\mathrm{s}}$ on $U$. We have solved this problem by introducing the method of interlaced pulses. The odd pulses have the function of a source of active particles and they are relatively long and high. The even pulses are used to measure the number of free electrons, produced by the previous breakdown. To minimize their influence, the measuring pulses are short and their voltage is low. When we fix the parameters $U_{1}$ and $T_{\mathrm{d} 1}$ of the source pulses, we can measure the dependence $P_{0}\left(U_{2}, T_{\mathrm{d} 2}, T_{\text {rep }}\right)$. When $P_{0}$ is kept constant by fixing $U_{2}$ and $T_{\mathrm{d} 2}$, the measurements of $I_{0}$ as a function of $U_{1}, T_{\mathrm{d} 1}$ and $T_{\text {rep }}$ are possible.

Figure 9 presents the formative time lag $t_{\mathrm{f} 2}$ of the measuring pulse as a function of the voltage $U_{2}$ of the measuring pulse, with the ionization kept constant by $1 \mathrm{~ms}$ source pulses of $1000 \mathrm{~V}$. The number of residing active particles was very high and the statistical delay time was reduced nearly to zero (in the graph, $t_{\mathrm{s}}$ is represented by the errorbars). In spite of large overvoltages $\left(U_{\mathrm{BR}} \approx 350 \mathrm{~V}\right)$ the following equation [21] holds

$$
\ln t_{\mathrm{f}}=c_{1}-c_{2} \ln \left(\frac{U-U_{\mathrm{BR}}}{U_{\mathrm{BR}}}\right) .
$$

The value of $c_{2}=2.8$ found in our case is much larger than the value for most gases, when conditions of the Townsend theory of breakdown are satisfied. Our results suggest that even for low $p d$ other processes, not accounted for by Townsend theory, may take place during a breakdown. Each series is measured twice but in a different order. This should suppress the mutual influence between the series (figure 7).

For extended discharge durations, the breakdown delay time is not reduced further but surprisingly it raises again, as

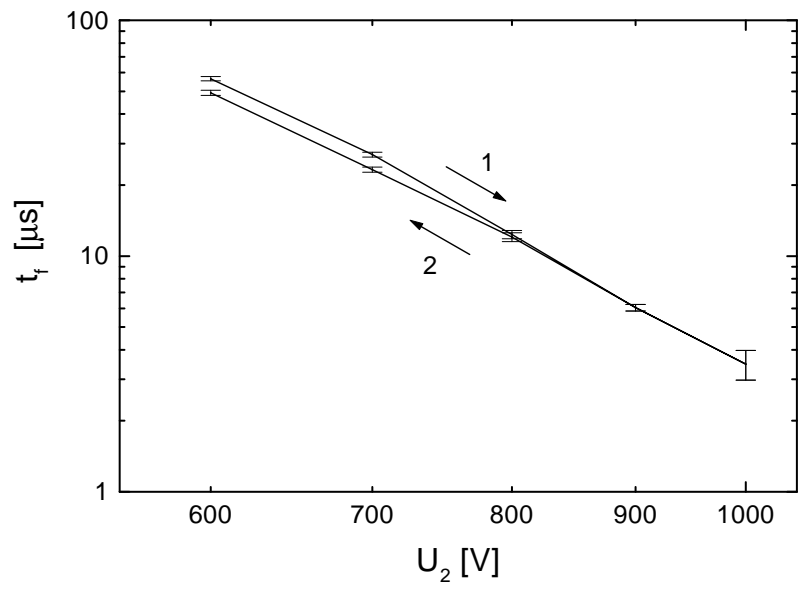

Figure 9. Formative time lag (line) and statistical delay time (errorbars) as functions of voltage. The constant ionization before each measuring pulse is generated by a source pulse with $U_{1}=1000 \mathrm{~V}$ and $T_{\mathrm{d} 1}=1 \mathrm{~ms}$. The other parameters are $T_{\text {rep }}=5 \mathrm{~s}, p=8$ Torr and $T_{\mathrm{d} 2}=20 \mu \mathrm{s}$.

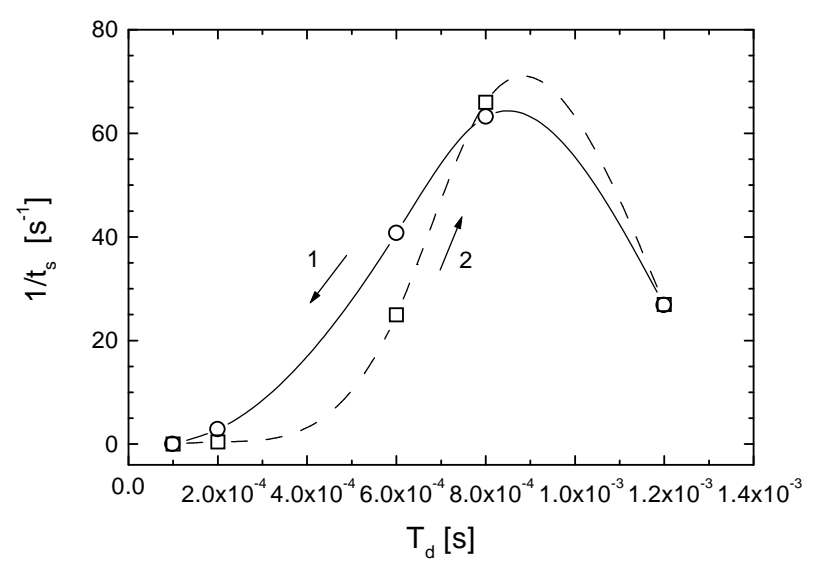

Figure 10. The quantity $1 / t_{\mathrm{s} 2}$, proportional to $I_{0} P_{0}$, is plotted versus discharge duration $T_{\mathrm{d} 1}$. To suppress the propagation of the memory effect from one series to the following, the value of $T_{\mathrm{d} 1}$ is first decreased from $1.2 \mathrm{~ms}$ down to $0.1 \mathrm{~ms}$ and then raised again. $U_{1}=1000 \mathrm{~V}, U_{2}=360 \mathrm{~V}, T_{\mathrm{d} 2}=30 \mu \mathrm{s}, T_{\text {rep }}=50 \mathrm{~s}$ and $p=4$ Torr.

shown in figure 10. This suggests that a process linked with the discharge has a hampering effect on the production of free electrons. It seems very improbable that the number of active particles decreases when $T_{\mathrm{d}}$ is prolongated. Obviously, $I_{0}$ and/or $P_{0}$ must decrease. This means that either the rate constant of a reaction, which produces free electrons from active species, is reduced or the discharge changes the probability $P_{0}$. The processes in the volume cannot last us until the next breakdown due to the flow of gas through the discharge tube. We deduce that surface processes are the cause of the observed memory effect and that discharges with long durations may change the state of the cathode or walls in a way which decreases $I_{0} P_{0}$.

Nearly all metals are covered with a thin layer of natural oxide. With insulating layer being charged by the ions and by the photoemission, the electric field in the dielectric may reach the value required for field emission [15]. When the leak and the emission currents are low, the layer may keep its charge for a very long time. To verify this hypothesis, 


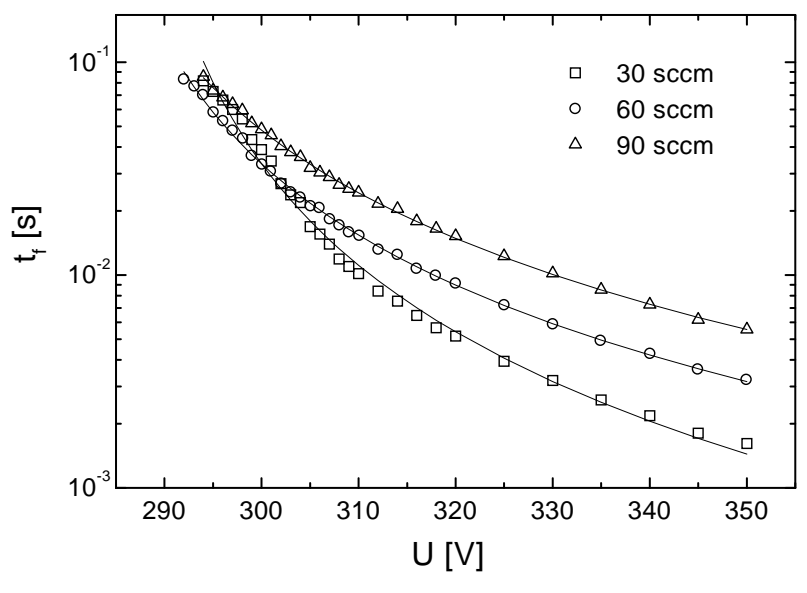

Figure 11. The formative time lag as a function of the voltage for low overvoltages plotted for three different gas flows: $p=4$ Torr, $T_{\mathrm{d}}=1 \mathrm{~ms}$ and $T_{\text {rep }}=0.2 \mathrm{~s}$.

we illuminated the whole discharge tube with a UV lamp and connected the sweep voltages in the range $(-200 \mathrm{~V}$; $+200 \mathrm{~V}$ ) to the electrodes during the period $T_{\text {rep }}$ between the measurements. Depending on the polarity of the sweep voltage, the charge on the surface of the insulating layer should be decreased or increased, which in turn should have an influence on the breakdown delay time of the next measurement. However, no such change was observed.

The same negative result was observed by the authors of [11]. They then concluded that the roughness of the metal surface is the cause of initiatory field emission. During the inactivity between measurements, the insulating layer grows due to the chemical reactions between the cathode material and the working gas or the impurities. Its higher work function and its smoothing effect on the protrusions and craters have an inhibiting influence on field emission. During the discharge, this layer is removed by ion bombardment and the field emission raises again. However, when the discharge burns for too long a time, the emissive irregularities on the surface may melt due to Joule heating and/or the impacts of positive ions.

Another mechanism was proposed for nitrogen in [14]. The nitrogen atoms created during the discharge adsorb to the cathode and then recombine due to its catalytic effect. The excited molecule of $N_{2}$ may then cause a release of the secondary electron from the cathode. In our case, no species capable of storing the energy for a long time are present with the exception of nitrogen impurities introduced by possible leaks. However, the concentration of nitrogen is in our case three orders of magnitude lower than in their experiments and yet our memory effects last for at least $10 \mathrm{~min}$.

If we look in detail [2] at the Townsend breakdown criterion from a statistical point of view, we see that the probability of one electron at the cathode producing an infinite series of avalanches is equal to zero for $U \leqslant U_{\mathrm{BR}}$ and rises with $U$ for $U>U_{\mathrm{BR}}$. Near the breakdown voltage, the probability $P_{0}$ is very low and thus the current $I_{0}$ of the initial electrons must be high to compensate for it. The real value of $U_{\mathrm{BR}}$ is unreachable, because even for $I_{0} \rightarrow \infty$ the statistical delay time will be infinite. However, in real experiments the period of waiting for a breakdown is always finite. Moreover, for very low overvoltages the formative time lag may also rise enormously. In figure 11 the dependence of $t_{\mathrm{f}}$ on voltage for low overvoltages is presented. Due to the high repetition rate $T_{\text {rep }}=0.2 \mathrm{~s}$, the statistical delay time may be neglected against $t_{\mathrm{f}}$. Measured data are fitted with equation (11) by the Marquardt-Levenberg method. All coefficients $c_{2}$ are close to $c_{2}=2$ which is higher than the value proposed in [21] $\left(c_{2}=1\right)$. As the best estimation of breakdown voltage we obtain $U_{\mathrm{BR}}(30 \mathrm{sccm})=285 \mathrm{~V}, U_{\mathrm{BR}}(60 \mathrm{sccm})=281 \mathrm{~V}$ and $U_{\mathrm{BR}}(90 \mathrm{sccm})=277 \mathrm{~V}$. These results were verified by the classical method and thus values presented here, being higher than those commonly found for $p d=8$ Torr $\mathrm{cm}$ for $\mathrm{He}$ gas and $\mathrm{Al}$ electrodes, should be probably attributed to non-degassed electrodes.

One may notice the contra-intuitive behaviour, where for the highest gas flow the breakdown voltage is the lowest one, but for voltages $15 \mathrm{~V}$ and more above the breakdown threshold the $t_{\mathrm{f}}$ is longer than for the other two curves. We can speculate that $P_{0}$ is higher and thus the breakdown voltage is shifted towards lower values. However, the higher gas flow may swerve the active particles so that they no longer reach the cathode, effectively reducing $I_{0}$. Changes of the gas flow mean also the changes of chemical composition of a gas when leaks exist. Also the pure mechanical effect of a gas stream may change the property of the adsorbate layer on a cathode.

\section{Conclusion}

The measurement of breakdown time delay is an efficient method for the investigation of lifetime of the active particles in the late afterglow. From the memory curves it is determined that a process, responsible for long-term creation of secondary electrons, is of second order. Some possible explanations are given and discussed. The volume processes are considered as non-influential due to the permanent gas flow through a discharge tube. The charging of insulating layers on the cathode is tested by small electronic or ionic currents, which should reduce or augment the surface charge. No influence on breakdown delay time is observed. The field emission from surface irregularities is a possible source of post emission, but it is not obvious why the insulating layer removal should be a second-order process. We consider a surface recombination of atoms and subsequent secondary electron emission as the most probable cause of post emission.

Fits of the dependences $t_{\mathrm{f}}=f(U)$ give higher values of the coefficient $c_{2}$ than is appropriate for a breakdown of Townsend type.

Besides a variation of the number of active particles, the discharge changes the state of the cathode, which has a pronounced influence on the delay of the next breakdown.

\section{Acknowledgments}

One of the authors (VK) was supported by Bourse de Gouvernement Français and by contract No 202/96/0508 of the Grant Agency of the Czech Republic.

\section{References}

[1] Schaefer G, Kristiansen M and Guenther A (eds) 1990 Gas Discharge Closing Switches vol 2 (Oxford: Plenum)

[2] Wijsman R A 1949 Phys. Rev. 75 833-8

[3] Legler W 1955 Z. Phys. 140 221-40 
[4] Legler W 1961 Z. Naturf. 16 253-61

[5] Davidson P M 1964 Proc. R. Soc. 83 259-73

[6] Evans C J 1987 J. Phys. D: Appl. Phys. 20 55-60

[7] Evans C J 1988 J. Phys. D: Appl. Phys. 21 1137-41

[8] Meek J M and Craags J D 1978 Electrical Breakdown of Gases (New York: Wiley)

[9] Suits C G and Hocker J P 1938 Phys. Rev. 53670

[10] Cobine J D 1938 Phys. Rev. 53911

[11] Woolsey G A and Ogle D B 1989 J. Appl. Phys. 66 2920-5

[12] Van Brunt R J and Cernyar E W 1991 Appl. Phys. Lett. 58 2628-30

[13] Aliaga R, Choi P and Kaufman Y 1992 Proc. 10th Int. Conf. on Gas Discharges and Their Applications (Swansea) vol 2, pp 788-91

[14] Marković V Lj, Pejović M M and Petrović Z Lj 1996 Plasma Chem. Plasma Process. 16 195-207
[15] Gavrilov N V and Mesyats G A 1997 Proc. ICPIG'97 (Toulouse) p II-32

[16] Agache M, Kudrle V, LeDuc E, Garcia P and Fitaire M 1994 Elementary Processes and Chemical Reactions in Low Temperature Plasma (Stará Lesná, Slovakia)

[17] Agache M, Kudrle V and Fitaire M 1994 Bull. Am. Phys. Soc. 396

[18] Agache M 1995 Modélisation de la phase d'amorçage d'une décharge électrique dans un gaz Doctoral Thesis Université Paris XI Orsay

[19] Kudrle V, LeDuc E and Fitaire M 1997 Proc. ICPIG'97 (Toulouse) p IV-28

[20] Loeb L B 1956 Handbuch der Physik XXI: Electrical Breakdown of Gases (Berlin: Springer)

[21] Maier II W B, Kadish A, Buchenauer C J and Robiscoe R T 1993 IEEE Trans. Plasma Sci. 21 676-83 